\title{
GENERAL WP-BAILEY CHAINS
}

\author{
JAMES MC LAUGHLIN AND PETER ZIMMER
}

\begin{abstract}
Motivated by a recent paper of Liu and Ma, we describe a number of general WP-Bailey chains. We show that many of the existing WP-Bailey chains (or branches of the WP-Bailey tree), including chains found by Andrews, Warnaar and Liu and Ma, arise as special cases of these general WP-Bailey chains.

We exhibit three new branches of the WP-Bailey tree, branches which also follow as special cases of these general WP-Bailey chains.

Finally, we describe a number of new transformation formulae for basic hypergeometric series which arise as consequences of these new WP-Bailey chains.
\end{abstract}

\section{INTRODUCTION}

Andrews [1], following on from prior work of Bressoud [4] and Singh [9], defined a WP-Bailey pair to be a pair of sequences $\left(\alpha_{n}(a, k, q), \beta_{n}(a, k, q)\right)$ (if the context is clear, we occasionally suppress the dependence on $q$ and write $\left.\left(\alpha_{n}(a, k), \beta_{n}(a, k)\right)\right)$ satisfying

$$
\begin{aligned}
\beta_{n}(a, k, q) & =\sum_{j=0}^{n} \frac{(k / a ; q)_{n-j}(k ; q)_{n+j}}{(q ; q)_{n-j}(a q ; q)_{n+j}} \alpha_{j}(a, k, q) \\
& =\frac{(k / a, k ; q)_{n}}{(a q, q ; q)_{n}} \sum_{j=0}^{n} \frac{\left(q^{-n} ; q\right)_{j}\left(k q^{n} ; q\right)_{j}}{\left(a q^{1-n} / k ; q\right)_{j}\left(a q^{n+1} ; q\right)_{j}}\left(\frac{q a}{k}\right)^{j} \alpha_{j}(a, k, q) .
\end{aligned}
$$

Andrews also showed in [1] that there were two distinct ways to construct new WP-Bailey pairs from a given pair (see (2.6) and (2.7) below). These two constructions allowed a "tree" of WP-Bailey pairs to be generated from a single WP-Bailey pair. Andrews and Berkovich [2] further investigated these two branches of the WP-Bailey tree, in the process deriving many new transformations for basic hypergeometric series. Spiridonov [10] derived an elliptic generalization of Andrews first WP-Bailey chain, and Warnaar [11] added four new branches to the WP-Bailey tree (see (2.8), (3.4), (3.5) and (3.11) below), two of which had generalizations to the elliptic level. More recently, and motivated in part by the papers above, Liu and Ma [7]

Date: January 18, 2019.

2000 Mathematics Subject Classification. Primary: 33D15. Secondary:11B65, 05A19.

Key words and phrases. Bailey chains, WP-Bailey Chains, WP-Bailey pairs. 
introduced the idea of a general WP-Bailey chain (as a solution to a system of linear equations), and added one new branch to the WP-Bailey tree (see (2.9) below).

Motivated by Liu and Ma's concept of a general WP-Bailey pair, in the present paper we reformulate their construction in terms of the more standard $(a, k)$ notation and also derive three other general WP-Bailey chains. We show that all the WP-Bailey chains referred to above arise as special cases of these general chains.

We further derive three new WP-Bailey chains from these general chains. Lastly, we find many new transformations of basic hypergeometric series, by inserting known WP-Bailey pairs into these three new WP-Bailey chains.

\section{The General WP-Bailey Chain of LiU and Ma}

Liu and Ma [7] defined a WP-Bailey chain as follows:

Definition 1. Any pair of sequences $\left\{\alpha_{n}(t, b)\right\}$ and $\left\{\beta_{n}(t, b)\right\}$ satisfying

$$
\beta_{n}(t, b)=\sum_{i=0}^{n} \frac{(b t ; q)_{n+i}(b ; q)_{n-i}}{(q ; q)_{n-i}(t q ; q)_{n+i}} \alpha_{i}(t, b)
$$

is called a well-poised (in short, WP) Bailey pair w.r.t. the parameters $t$ and $b$, denoted by $\left(\alpha_{n}(t, b), \beta_{n}(t, b)\right)$. Further, if for $n \geq 0$, the sequences $\left\{\alpha_{n}^{\prime}(t, c)\right\}$ and $\left\{\beta_{n}^{\prime}(t, c)\right\}$ defined by

$$
\begin{aligned}
& \alpha_{n}^{\prime}(t, c)=\lambda_{n} \alpha_{n}(t, b), \\
& \beta_{n}^{\prime}(t, c)=\sum_{i=0}^{n} d_{n, i} \beta_{i}(t, b)
\end{aligned}
$$

is still a WP-Bailey pair w.r.t. the parameters $t$ and $c$, then the iterative process from $\left(\alpha_{n}(t, b), \beta_{n}(t, b)\right)$ to $\left(\alpha_{n}^{\prime}(t, c), \beta_{n}^{\prime}(t, c)\right)$ is said to be a WP-Bailey chain.

The authors point out in [7] that the $\lambda_{n}$ and the $d_{n, i}$ always satisfy

$$
\sum_{i=k}^{n} \frac{(b t ; q)_{i+k}(b ; q)_{i-k}}{(q ; q)_{i-k}(t q ; q)_{i+k}} d_{n, i}=\frac{(c t ; q)_{n+k}(c ; q)_{n-k}}{(q ; q)_{n-k}(t q ; q)_{n+k}} \lambda_{k}
$$

and then proceed, using infinite matrices, to invert (2.3) to find expressions for the $d_{n, k}$ as sums over the $\lambda_{i}$.

For our purposes, we reformulate their result using the more usual notation in which $\left(\alpha_{n}(a, k), \beta_{n}(a, k)\right)$ denotes a WP-Bailey pair, using a single sequence $g_{n}$ (replacing their $\lambda_{n}$ ), and giving a more direct proof which avoids the use of infinite matrices. Before proceeding to this, we first recall the following necessary result of Warnaar. 
Lemma 1 (Warnaar, 11]). For a and $k$ indeterminates the following equations are equivalent:

$$
\begin{aligned}
& \beta_{n}(a, k)=\sum_{j=0}^{n} \frac{(k / a)_{n-j}(k)_{n+j}}{(q)_{n-j}(a q)_{n+j}} \alpha_{j}(a, k), \\
& \alpha_{n}(a, k)=\frac{1-a q^{2 n}}{1-a} \sum_{j=0}^{n} \frac{1-k q^{2 j}}{1-k} \frac{(a / k)_{n-j}(a)_{n+j}}{(q)_{n-j}(k q)_{n+j}}\left(\frac{k}{a}\right)^{n-j} \beta_{j}(a, k) .
\end{aligned}
$$

If $a$ and $k$ are interchanged in the second equation, we also note for later that Lemma 1 implies the following.

Corollary 1. If $\left(\alpha_{n}(a, k), \beta_{n}(a, k)\right)$ are a WP-Bailey pair, then so are $\left(\alpha_{n}^{\prime}(a, k), \beta_{n}^{\prime}(a, k)\right)$, where

$$
\begin{aligned}
& \alpha_{n}^{\prime}(a, k)=\frac{1-a q^{2 n}}{1-a}\left(\frac{k}{a}\right)^{n} \beta_{n}(k, a), \\
& \beta_{n}^{\prime}(a, k)=\frac{1-k}{1-k q^{2 n}}\left(\frac{k}{a}\right)^{n} \alpha_{n}(k, a) .
\end{aligned}
$$

Theorem 1. Suppose that $\left(\alpha_{n}(a, k), \beta_{n}(a, k)\right)$ satisfy (1.1), that $g_{n}$ is an arbitrary sequence of functions and $c$ and $e$ are arbitrary constants. Then $\left(\alpha_{n}^{\prime}(a, k), \beta_{n}^{\prime}(a, k)\right)$ also satisfy (1.1), where

$$
\begin{aligned}
& \alpha_{n}^{\prime}(a, k)=g_{n} \alpha_{n}(e, c), \\
& \beta_{n}^{\prime}(a, k)=\sum_{j=0}^{n} \beta_{j}(e, c) \frac{\left(1-c q^{2 j}\right)(k / a ; q)_{n-j}(k ; q)_{n+j}(e q ; q)_{2 j}}{(1-c)(q ; q)_{n-j}(a q ; q)_{n+j}(c q ; q)_{2 j}} \\
& \quad \times \sum_{r=0}^{n-j} \frac{\left(1-e q^{2 j+2 r}\right)\left(q^{-(n-j)}, k q^{n+j}, e / c, e q^{2 j} ; q\right)_{r}}{\left(1-e q^{2 j}\right)\left(a q^{n+j+1}, a q^{1-(n-j)} / k, c q^{2 j+1}, q ; q\right)_{r}}\left(\frac{q a c}{k e}\right)^{r} g_{r+j} .
\end{aligned}
$$

Proof. If $\alpha_{n}^{\prime}(a, k)$ is as given, then

$$
\begin{aligned}
& \beta_{n}^{\prime}(a, k)= \sum_{r=0}^{n} \frac{(k / a)_{n-r}(k)_{n+r}}{(q)_{n-r}(a q)_{n+r}} g_{r} \alpha_{r}(e, c) \\
&= \sum_{r=0}^{n} \frac{(k / a)_{n-r}(k)_{n+r}}{(q)_{n-r}(a q)_{n+r}} g_{r} \\
& \quad \times \frac{1-e q^{2 r}}{1-e} \sum_{j=0}^{r} \frac{1-c q^{2 j}}{1-c} \frac{(e / c)_{r-j}(e)_{r+j}}{(q)_{r-j}(c q)_{r+j}}\left(\frac{c}{e}\right)^{r-j} \beta_{j}(e, c) \\
&= \sum_{j=0}^{n} \frac{1-c q^{2 j}}{1-c} \beta_{j}(e, c) \\
& \quad \times \sum_{r=j}^{n} \frac{(k / a)_{n-r}(k)_{n+r}}{(q)_{n-r}(a q)_{n+r}} g_{r} \frac{1-e q^{2 r}}{1-e} \frac{(e / c)_{r-j}(e)_{r+j}}{(q)_{r-j}(c q)_{r+j}}\left(\frac{c}{e}\right)^{r-j}
\end{aligned}
$$




$$
\begin{aligned}
= & \sum_{j=0}^{n} \frac{1-c q^{2 j}}{1-c} \beta_{j}(e, c) \\
& \times \sum_{r=0}^{n-j} \frac{(k / a)_{n-r-j}(k)_{n+r+j}}{(q)_{n-r-j}(a q)_{n+r+j}} g_{r+j} \frac{1-e q^{2 r+2 j}}{1-e} \frac{(e / c)_{r}(e)_{r+2 j}}{(q)_{r}(c q)_{r+2 j}}\left(\frac{c}{e}\right)^{r} \\
= & \sum_{j=0}^{n} \frac{1-c q^{2 j}}{1-c} \beta_{j}(e, c) \frac{(k / a)_{n-j}(k)_{n+j}(e q)_{2 j}}{(q)_{n-j}(a q)_{n+j}(c q)_{2 j}} \\
\times & \sum_{r=0}^{n-j} \frac{1-e q^{2 r+2 j}}{1-e q^{2 j}} \frac{\left(q^{-(n-j)}, k q^{n+j}, e / c, e q^{2 j} ; q\right)_{r}}{\left(a q^{1-(n-j)} / k, a q^{n+j+1}, c q^{2 j+1}, q ; q\right)_{r}}\left(\frac{q a c}{k e}\right)^{r} g_{r+j} .
\end{aligned}
$$

Of course, from the perspective of adding branches to the WP-Bailey tree, what is desirable is to choose $e$ and $c$ and the sequence $g_{n}$ so as to make the inner sum over $r$ above have closed form.

It is shown in [7] that Andrews' first WP-Bailey chain in [1] follows as a special case of their theorem, and the authors also find a new WP-Bailey chain as another special case. In fact Andrews' second WP-Bailey chain in [1] and the first WP-Bailey chain of Warnaar in [11] also follow as special cases. Since our notation is somewhat different from that of Liu and Ma [7], for completeness we show how all four WP Bailey chains follow as special cases of Theorem 1 .

Corollary 2. If $\left(\alpha_{n}(a, k), \beta_{n}(a, k)\right)$ satisfy (1.1), then so do $\left(\alpha_{n}^{\prime}(a, k)\right.$, $\left.\beta_{n}^{\prime}(a, k)\right)$ and $\left(\tilde{\alpha}_{n}(a, k), \tilde{\beta}_{n}(a, k)\right)$ [1], $\left(\alpha_{n}^{\dagger}(a, k), \beta_{n}^{\dagger}(a, k)\right)$ [1] and $\left(\alpha_{n}^{*}(a, k)\right.$, $\left.\beta_{n}^{*}(a, k)\right)$ 7], where

$$
\begin{aligned}
\alpha_{n}^{\prime}(a, k) & =\frac{\left(\rho_{1}, \rho_{2}\right)_{n}}{\left(a q / \rho_{1}, a q / \rho_{2}\right)_{n}}\left(\frac{a q}{\rho_{1} \rho_{2}}\right)^{n} \alpha_{n}(a, c), \quad(\text { Andrews [1] }) \\
\beta_{n}^{\prime}(a, k) & =\frac{\left(k \rho_{1} / a, k \rho_{2} / a\right)_{n}}{\left(a q / \rho_{1}, a q / \rho_{2}\right)_{n}} \times \\
& \sum_{j=0}^{n} \frac{\left(1-c q^{2 j}\right)\left(\rho_{1}, \rho_{2}\right)_{j}(k / c)_{n-j}(k)_{n+j}}{(1-c)\left(k \rho_{1} / a, k \rho_{2} / a\right)_{n}(q)_{n-j}(q c)_{n+j}}\left(\frac{a q}{\rho_{1} \rho_{2}}\right)^{j} \beta_{j}(a, c),
\end{aligned}
$$

with $c=k \rho_{1} \rho_{2} / a q$;

$$
\begin{aligned}
& \left.\tilde{\alpha}_{n}(a, k)=\frac{\left(q a^{2} / k\right)_{2 n}}{(k)_{2 n}}\left(\frac{k^{2}}{q a^{2}}\right)^{n} \alpha_{n}(a, c), \quad \text { (Andrews [1] }\right) \\
& \tilde{\beta}_{n}(a, k)=\sum_{j=0}^{n} \frac{\left(k^{2} / q a^{2}\right)_{n-j}}{(q)_{n-j}}\left(\frac{k^{2}}{q a^{2}}\right)^{j} \beta_{j}(a, c)
\end{aligned}
$$

with $c=q a^{2} / k$;

(Warnaar [1]) 


$$
\begin{aligned}
& \alpha_{n}^{\dagger}(a, k)=\frac{1-k^{1 / 2}}{1-q^{n} k^{1 / 2}} \frac{1+q^{n} a / k^{1 / 2}}{1+a / k^{1 / 2}} \frac{\left(a^{2} / k ; q\right)_{2 n}}{(k ; q)_{2 n}}\left(\frac{k^{2}}{a^{2}}\right)^{n} \alpha_{n}(a, c), \\
& \beta_{n}^{\dagger}(a, k)=\frac{1-k^{1 / 2}}{1-q^{n} k^{1 / 2}} \sum_{j=0}^{n} \frac{1+q^{j} a / k^{1 / 2}}{1+a / k^{1 / 2}} \frac{(k / c ; q)_{n-j}}{(q ; q)_{n-j}}\left(\frac{k^{2}}{a^{2}}\right)^{j} \beta_{j}(a, c),
\end{aligned}
$$

with $c=a^{2} / k$ (we consider just the $\sigma=1$ case of Warnaar's theorem here);

$$
\begin{aligned}
& \alpha_{n}^{*}(a, k)=\frac{\left(a^{2} / k ; q^{2}\right)_{n}}{\left(k q ; q^{2}\right)_{n}}\left(\frac{-k}{a}\right)^{n} \alpha_{n}(a, c), \\
& \beta_{n}^{*}(a, k)=\sum_{j=0}^{\lfloor n / 2\rfloor} \frac{1-\frac{a^{2} q^{2 n-4 j}}{k}}{1-\frac{a^{2}}{k}} \frac{\left(k ; q^{2}\right)_{n-j}\left(k^{2} / a^{2} ; q^{2}\right)_{j}}{\left(a^{2} q^{2} / k ; q^{2}\right)_{n-j}\left(q^{2} ; q^{2}\right) j}\left(\frac{-k}{a}\right)^{n-2 j} \beta_{n-2 j}(a, c),
\end{aligned}
$$

with $c=a^{2} / k$.

Proof. In each case we apply Theorem 1, letting $e=a$ and $g_{n}$ be the factor multiplying $\alpha_{n}(a, c)$ in each of the WP-Bailey chains listed above. That the inner sum over $r$ in (2.5) has a closed form and that the stated expressions for the various $\beta_{n}^{\text {new }}(a, k)$ above now hold, follow as a consequence of, respectively, Jackson's sum of a terminating ${ }_{8} \phi_{7}$,

$$
\begin{array}{r}
{ }_{8} \phi_{7}\left[\begin{array}{r}
a, q a^{1 / 2},-q a^{1 / 2}, b, c, d, e, q^{-n} \\
a^{1 / 2},-a^{1 / 2}, a q / b, a q / c, a q / d
\end{array}, a q / e, a q^{n+1} ; q, q\right] \\
=\frac{(a q, a q / b c, a q / b d, a q / c d ; q)_{n}}{(a q / b, a q / c, a q / d, a q / b c d ; q)_{n}}
\end{array}
$$

where $a^{2} q=b c d e q^{-n}$, the following formulae (see [6, problem 2.12] and [2, Equation (3.2)], respectively):

$$
\begin{array}{r}
{ }_{10} \phi_{9}\left[\begin{array}{r}
\left.a, q \sqrt{a},-q \sqrt{a}, a \sqrt{\frac{q}{k}},-a \sqrt{\frac{q}{k}}, \frac{a q}{\sqrt{k}},-\frac{a q}{\sqrt{k}}, \frac{k}{a q}, k q^{n}, q^{-n} ; q, q\right] \\
\left.\sqrt{a},-\sqrt{a}, \sqrt{k q},-\sqrt{k q}, \sqrt{k},-\sqrt{k}, \frac{a^{2} q^{2}}{k}, \frac{a q^{1-n}}{k}, a q^{n+1}\right] \\
=\frac{\left(a q, \frac{k^{2}}{q a^{2}} ; q\right)_{n}}{\left(k, \frac{k}{a} ; q\right)_{n}} \\
{ }_{10} \phi_{9}\left[\begin{array}{l}
\left.a, q \sqrt{a},-q \sqrt{a}, a \sqrt{\frac{q}{k}},-a \sqrt{\frac{q}{k}}, \frac{a}{\sqrt{k}},-\frac{a q}{\sqrt{k}}, \frac{k}{a}, k q^{n}, q^{-n} ; q, q\right] \\
\sqrt{a},-\sqrt{a}, \sqrt{k q},-\sqrt{k q}, q \sqrt{k},-\sqrt{k}, \frac{a^{2} q}{k}, \frac{a q^{1-n}}{k}, a q^{n+1}
\end{array}\right] \\
=\frac{\left(a q, \sqrt{k}, \frac{k^{2}}{a^{2}} ; q\right)_{n}}{\left(k, \frac{k}{a}, q \sqrt{k} ; q\right)_{n}}
\end{array}\right.
\end{array}
$$


and a $q$-analogue of Watson's ${ }_{3} F_{2}$ sum (see [6, II.16]),

$$
\begin{aligned}
{ }_{8} \phi_{7}\left[\begin{array}{cc}
\lambda, q \sqrt{\lambda},-q \sqrt{\lambda}, q^{-n}, b q^{n}, \lambda \sqrt{q / b},-\lambda \sqrt{q / b}, b / \lambda \\
\left.\sqrt{\lambda},-\sqrt{\lambda}, \lambda q^{n+1}, \lambda q^{1-n} / b, \lambda^{2} q / b, \sqrt{q b},-\sqrt{q b} ; q,-\frac{q \lambda}{b}\right] & n \text { odd }
\end{array}\right. \\
\quad= \begin{cases}0, & n \text { even. } \\
\frac{(\lambda q ; q)_{n}}{(b / \lambda ; q)_{n}} \frac{\left(q, b^{2} / \lambda^{2} ; q^{2}\right)_{n / 2}}{\left(b q, q^{2} \lambda^{2} / b ; q^{2}\right)_{n / 2}}, & \end{cases}
\end{aligned}
$$

Before describing and proving a new WP-Bailey chain which follows from Theorem 1, we need a preliminary result.

\section{Lemma 2.}

$$
\begin{array}{r}
{ }_{10} \phi_{9}\left[\begin{array}{r}
a, q \sqrt{a},-q \sqrt{a}, a \sqrt{\frac{q}{k}},-a \sqrt{\frac{q}{k}}, \frac{a}{\sqrt{k}},-\frac{a}{\sqrt{k}}, \frac{k q}{a}, k q^{n}, q^{-n} \\
\sqrt{a},-\sqrt{a}, \sqrt{k q},-\sqrt{k q}, q \sqrt{k},-q \sqrt{k}, \frac{a^{2}}{k}, \frac{a q^{1-n}}{k}, a q^{n+1}
\end{array}\right] \\
=\frac{1-k}{1-k q^{2 n}} \frac{\left(a q, \frac{k^{2} q}{a^{2}} ; q\right)_{n}}{\left(k, \frac{k}{a} ; q\right)_{n}} .
\end{array}
$$

Proof. In Bailey's transformation (see [6], III.28),

$$
\begin{gathered}
\text { (2.14) }{ }_{10} \phi_{9}\left[\begin{array}{c}
a, q \sqrt{a},-q \sqrt{a}, b, c, d, e, f, \lambda a q^{n+1} / e f, q^{-n} \\
\left.\sqrt{a},-\sqrt{a}, a q / b, a q / c, a q / d, a q / e, a q / f, e f q^{-n} / \lambda, a q^{n+1} ; q, q\right]
\end{array}\right] \\
=\frac{(a q, a q / e f, \lambda q / e, \lambda q / f ; q)_{n}}{(a q / e, a q / f, \lambda q / e f, \lambda q ; q)_{n}} \\
\times{ }_{10} \phi_{9}\left[\begin{array}{c}
\lambda, q \sqrt{\lambda},-q \sqrt{\lambda}, \lambda b / a, \lambda c / a, \lambda d / a, e, f, \lambda a q^{n+1} / e f, q^{-n} \\
\left.\sqrt{\lambda},-\sqrt{\lambda}, a q / b, a q / c, a q / d, \lambda q / e, \lambda q / f, e f q^{-n} / a, \lambda q^{n+1} ; q, q\right]
\end{array}\right.
\end{gathered}
$$

where $\lambda=q a^{2} / b c d$, set $b=a \sqrt{q / k}, c=-a \sqrt{q / k}, d=k / a q, e=a q / \sqrt{k}$ and $f=-a q / \sqrt{k}$, so that $\lambda=-a q$. The left side of (2.14) becomes the left side of (2.11), and so equals the right side of (2.11). The result follows upon replacing $a$ with $-a / q$, after some simple manipulations.

The next WP-Bailey chain appears to be new.

Corollary 3. If $\left(\alpha_{n}(a, k, q), \beta_{n}(a, k, q)\right)$ satisfy (1.1), then so do $\left(\alpha_{n}^{\prime}(a, k, q)\right.$, $\left.\beta_{n}^{\prime}(a, k, q)\right)$, where

$$
\begin{aligned}
& \alpha_{n}^{\prime}(a, k, q)=\frac{\left(\frac{a^{2}}{k} ; q\right)_{2 n}}{(k q ; q)_{2 n}}\left(\frac{k^{2} q}{a^{2}}\right)^{n} \alpha_{n}\left(a, \frac{a^{2}}{k q}, q\right), \\
& \beta_{n}^{\prime}(a, k, q)=\frac{1-k}{1-k q^{2 n}} \sum_{j=0}^{n} \frac{\left(1-\frac{a^{2} q^{2 j}}{k q}\right)}{\left(1-\frac{a^{2}}{k q}\right)} \frac{\left(\frac{k^{2} q}{a^{2}} ; q\right)_{n-j}}{(q ; q)_{n-j}}\left(\frac{k^{2} q}{a^{2}}\right)^{j} \beta_{j}\left(a, \frac{a^{2}}{k q}, q\right) .
\end{aligned}
$$


Proof. Set $e=a, c=a^{2} / k q, g_{n}=\left(k^{2} q / a^{2}\right)^{n}\left(a^{2} / k ; q\right)_{2 n} /(k q ; q)_{2 n}$ in Theorem 1, and use (2.13), with $a$ replaced with $a q^{2 j}$ and $k$ with $k q^{2 j}$, to put the inner sum over $r$ at (2.5) in closed form, and simplify the resulting sum.

Remarks: 1) We note the similarities between this WP-Bailey chain and the second chain of Andrews at (2.7), including the fact that a double application of this construction returns the original WP-Bailey pair.

2) Upon inserting the unit WP-Bailey pair (4.1) in this chain, we get the pair

$$
\begin{aligned}
\alpha_{n}(a, k) & =\frac{1-a q^{2 n}}{1-a} \frac{(a, k q / a ; q)_{n}}{\left(q, a^{2} / k ; q\right)_{n}} \frac{\left(a^{2} / k ; q\right)_{2 n}}{(k q ; q)_{2 n}}\left(\frac{k}{a}\right)^{n}, \\
\beta_{n}(a, q) & =\frac{1-k}{1-k q^{2 n}} \frac{\left(q k^{2} / a^{2} ; q\right)_{n}}{(q ; q)_{n}}
\end{aligned}
$$

which can also be derived by applying Corollary 1 to the pair at (4.14).

3) The small number of summation formulae for terminating basic hypergeometric series would tend to suggest that there are not many WP-Bailey chains which follow from Theorem 1. With this in mind, we turn to new general WP-Bailey chains.

\section{New General WP-Bailey Chains and their Consequences}

Motivated by Warnaar's other WP-Bailey chains in [11, we now consider some new general WP-Bailey chains.

Theorem 2. Suppose that $\left(\alpha_{n}(a, k, q), \beta_{n}(a, k, q)\right)$ satisfy (1.1), that $g_{n}$ is an arbitrary sequence of functions and that $e$ and $c$ are arbitrary constants. Then $\left(\alpha_{n}^{\prime}(a, k, q), \beta_{n}^{\prime}(a, k, q)\right)$ also satisfy (1.1), where

$$
\begin{aligned}
& \alpha_{n}^{\prime}(a, k, q)=g_{n} \alpha_{n}\left(e, c, q^{2}\right), \\
& \beta_{n}^{\prime}(a, k, q)=\sum_{j=0}^{n} \beta_{j}\left(e, c, q^{2}\right) \frac{\left(1-c q^{4 j}\right)(k / a ; q)_{n-j}(k ; q)_{n+j}\left(e q^{2} ; q^{2}\right)_{2 j}}{(1-c)(q ; q)_{n-j}(a q ; q)_{n+j}\left(c q^{2} ; q^{2}\right)_{2 j}} \\
& \times \sum_{r=0}^{n-j} \frac{\left(1-e q^{4 j+4 r}\right)\left(q^{-(n-j)}, k q^{n+j} ; q\right)_{r}\left(e / c, e q^{4 j} ; q^{2}\right)_{r} g_{r+j}}{\left(1-e q^{4 j}\right)\left(a q^{n+j+1}, a q^{1-(n-j)} / k ; q\right)_{r}\left(c q^{4 j+2}, q^{2} ; q^{2}\right)_{r}}\left(\frac{q c a}{k e}\right)^{r} .
\end{aligned}
$$

Proof. As previously, if $\alpha_{n}^{\prime}(a, k, q)$ is as given, then

$$
\begin{aligned}
\beta_{n}^{\prime}(a, k, q) & =\sum_{r=0}^{n} \frac{(k / a)_{n-r}(k)_{n+r}}{(q)_{n-r}(a q)_{n+r}} g_{r} \alpha_{r}\left(e, c, q^{2}\right) \\
& =\sum_{r=0}^{n} \frac{(k / a)_{n-r}(k)_{n+r}}{(q)_{n-r}(a q)_{n+r}} g_{r} \\
& \times \frac{1-e q^{4 r}}{1-e} \sum_{j=0}^{r} \frac{1-c q^{4 j}}{1-c} \frac{\left(e / c ; q^{2}\right)_{r-j}\left(e ; q^{2}\right)_{r+j}}{\left(q^{2} ; q^{2}\right)_{r-j}\left(c q^{2} ; q^{2}\right)_{r+j}}\left(\frac{c}{e}\right)^{r-j} \beta_{j}\left(e, c, q^{2}\right)
\end{aligned}
$$




$$
\begin{aligned}
& =\sum_{j=0}^{n} \frac{1-c q^{4 j}}{1-c} \beta_{j}\left(e, c, q^{2}\right) \\
& \times \sum_{r=j}^{n} \frac{(k / a)_{n-r}(k)_{n+r}}{(q)_{n-r}(a q)_{n+r}} g_{r} \frac{1-e q^{4 r}}{1-a} \frac{\left(e / c ; q^{2}\right)_{r-j}\left(e ; q^{2}\right)_{r+j}}{\left(q^{2} ; q^{2}\right)_{r-j}\left(c q^{2} ; q^{2}\right)_{r+j}}\left(\frac{c}{e}\right)^{r-j} \\
& =\sum_{j=0}^{n} \frac{1-c q^{4 j}}{1-c} \beta_{j}\left(e, c, q^{2}\right) \sum_{r=0}^{n-j} \frac{(k / a)_{n-r-j}(k)_{n+r+j}}{(q)_{n-r-j}(a q)_{n+r+j}} \\
& \quad \times g_{r+j} \frac{1-e q^{4 r+4 j}}{1-e} \frac{\left(e / c ; q^{2}\right)_{r}\left(e ; q^{2}\right)_{r+2 j}}{\left(q^{2} ; q^{2}\right)_{r}\left(c q^{2} ; q^{2}\right)_{r+2 j}}\left(\frac{c}{e}\right)^{r} \\
& =\sum_{j=0}^{n} \frac{1-c q^{4 j}}{1-c} \beta_{j}\left(e, c, q^{2}\right) \frac{(k / a)_{n-j}(k)_{n+j}\left(e q^{2} ; q^{2}\right)_{2 j}}{(q)_{n-j}(a q)_{n+j}\left(c q^{2} ; q^{2}\right)_{2 j}} \sum_{r=0}^{n-e} \frac{1-e q^{4 r+4 j}}{1-e q^{4 j}} \\
& \quad \times \frac{\left(q^{-(n-j)}, k q^{n+j} ; q\right)_{r}\left(e / c, e q^{4 j} ; q^{2}\right)_{r}}{\left(a q^{1-(n-j)} / k, a q^{n+j+1} ; q\right)_{r}\left(c q^{4 j+2}, q^{2} ; q^{2}\right)_{r}}\left(\frac{q a c}{k e}\right)^{r} g_{r+j} .
\end{aligned}
$$

As with Theorem 1, the aim is to find choices for the sequence $g_{n}$ and the parameters $e$ and $c$ which lead to the inner sum over $r$ above having closed form. We believe the following WP-Bailey chain to be new.

Corollary 4. If $\left(\alpha_{n}(a, k, q), \beta_{n}(a, k, q)\right)$ satisfy (1.1), then so do $\left(\alpha_{n}^{\prime}(a, k, q)\right.$, $\left.\beta_{n}^{\prime}(a, k, q)\right)$, where

$$
\begin{aligned}
& \alpha_{n}^{\prime}(a, k, q)= \frac{1+a}{1+a q^{2 n}} q^{n} \alpha_{n}\left(a^{2}, \frac{a k}{q}, q^{2}\right) \\
& \beta_{n}^{\prime}(a, k, q)=\sum_{j=0}^{n} \frac{\left(1-\frac{a k}{q} q^{4 j}\right)}{\left(1-\frac{a k}{q}\right)} \frac{(-a q ; q)_{2 j}}{(-k ; q)_{2 j}} \frac{\left(\frac{q k}{a} ; q^{2}\right)_{n-j}}{\left(q^{2} ; q^{2}\right)_{n-j}} \frac{\left(k^{2} ; q^{2}\right)_{n+j}}{\left(a k q ; q^{2}\right)_{n+j}} \\
& \times \frac{1+a}{1+a q^{2 j}} q^{j} \beta_{j}\left(a^{2}, \frac{a k}{q}, q^{2}\right) .
\end{aligned}
$$

Proof. Set $e=a^{2}, c=a k / q, g_{n}=(1+a) q^{n} /\left(1+a q^{2 n}\right)$ in Theorem 2, and use (2.10) to put the inner sum over $r$ at (3.1) in closed form, and simplify the resulting sum.

Remark: Somewhat curiously, inserting the unit pair (4.1) in this chain leads to a pair that is the special case $\rho_{1}=-\rho_{2}=\sqrt{a q / k}$ of Singh's WPBailey pair at (4.2).

In Theorem 3 we showed how to derive new general WP-Bailey pairs $\left(\alpha_{n}^{\prime}(a, k, q), \beta_{n}^{\prime}(a, k, q)\right)$ in terms of $\left(\alpha_{n}\left(e, c, q^{2}\right), \beta_{n}\left(e, c, q^{2}\right)\right)$, where $\left(\alpha_{n}(a, k, q), \beta_{n}(a, k, q)\right)$ is an existing WP-Bailey pair. It is also possible to derive new general WP-Bailey pairs in terms of $\left(\alpha_{n}\left(e, c, q^{1 / 2}\right), \beta_{n}\left(e, c, q^{1 / 2}\right)\right)$, 
but for aesthetic reasons we replace $q^{1 / 2}$ by $q$ everywhere, to get the following theorem.

Theorem 3. Suppose that $\left(\alpha_{n}(a, k, q), \beta_{n}(a, k, q)\right)$ satisfy (1.1), that $g_{n}$ is an arbitrary sequence of functions and that $e$ and $c$ are arbitrary constants. Then $\left(\alpha_{n}^{\prime}(a, k, q), \beta_{n}^{\prime}(a, k, q)\right)$ also satisfy (1.1), where

$$
\begin{aligned}
\alpha_{n}^{\prime}\left(a, k, q^{2}\right) & =g_{n} \alpha_{n}(e, c, q), \\
\beta_{n}^{\prime}\left(a, k, q^{2}\right) & =\sum_{j=0}^{n} \beta_{j}(e, c, q) \frac{\left(1-c q^{2 j}\right)\left(k / a ; q^{2}\right)_{n-j}\left(k ; q^{2}\right)_{n+j}(e q ; q)_{2 j}}{(1-c)\left(q^{2} ; q^{2}\right)_{n-j}\left(a q^{2} ; q^{2}\right)_{n+j}(c q ; q)_{2 j}} \\
\times \sum_{r=0}^{n-j} & \frac{\left(1-e q^{2 j+2 r}\right)\left(q^{-2(n-j)}, k q^{2 n+2 j} ; q^{2}\right)_{r}\left(e / c, e q^{2 j} ; q\right)_{r} g_{r+j}}{\left(1-e q^{2 j}\right)\left(a q^{2 n+2 j+2}, a q^{2-2(n-j)} / k ; q^{2}\right)_{r}\left(c q^{2 j+1}, q ; q\right)_{r}}\left(\frac{q^{2} c a}{k e}\right)^{r} .
\end{aligned}
$$

We omit the proof as it follows very similar lines to the proofs of Theorems 1] and 2, Warnaar's WP-Bailey chains in Theorems 2.4 and 2.5 of [11] follows as a special case of the theorem above (we use $\sqrt{a}$ where Warnaar uses $a$, so as to keep the parameters in the new WP Bailey pairs as $a$ and $k$ ).

Corollary 5 (Warnaar, [1]). If $\left(\alpha_{n}(a, k, q), \beta_{n}(a, k, q)\right)$ satisfy (1.1), then so do $\left(\alpha_{n}^{\prime}(a, k, q), \beta_{n}^{\prime}(a, k, q)\right)$, where

$$
\begin{aligned}
\alpha_{n}^{\prime}\left(a, k, q^{2}\right)= & \alpha_{n}\left(\sqrt{a}, \frac{k}{q \sqrt{a}}, q\right) \\
\beta_{n}^{\prime}\left(a, k, q^{2}\right)= & \frac{\left(\frac{-k}{\sqrt{a}} ; q\right)_{2 n}}{(-q \sqrt{a} ; q)_{2 n}} \sum_{j=0}^{n} \frac{\left(1-\frac{k q^{2 j}}{q \sqrt{a}}\right)}{\left(1-\frac{k}{q \sqrt{a}}\right)} \frac{\left(\frac{a q^{2}}{k} ; q^{2}\right)_{n-j}}{\left(q^{2} ; q^{2}\right)_{n-j}} \frac{\left(k ; q^{2}\right)_{n+j}}{\left(\frac{k^{2}}{a} ; q^{2}\right)_{n+j}} \\
& \times\left(\frac{k}{a q}\right)^{n-j} \beta_{j}\left(\sqrt{a}, \frac{k}{q \sqrt{a}}, q\right) .
\end{aligned}
$$

Proof. Set $e=\sqrt{a}, c=k / q \sqrt{a}, g_{n}=1$, use (2.10) to get the inner sum over $r$ in (3.3) in closed form, and the result follows after some simple manipulations.

Corollary 6 (Warnaar, [11]). If $\left(\alpha_{n}(a, k, q), \beta_{n}(a, k, q)\right)$ satisfy (1.1), then so do $\left(\alpha_{n}^{\prime}(a, k, q), \beta_{n}^{\prime}(a, k, q)\right)$, where

$$
\begin{aligned}
& \alpha_{n}^{\prime}\left(a, k, q^{2}\right)=q^{-n} \frac{1+\sqrt{a} q^{2 n}}{1+\sqrt{a}} \alpha_{n}\left(\sqrt{a}, \frac{k}{\sqrt{a}}, q\right) \\
& \beta_{n}^{\prime}\left(a, k, q^{2}\right)=q^{-n} \frac{\left(\frac{-k q}{\sqrt{a}} ; q\right)_{2 n}}{(-\sqrt{a} ; q)_{2 n}} \sum_{j=0}^{n} \frac{\left(1-\frac{k q^{2 j}}{\sqrt{a}}\right)}{\left(1-\frac{k}{\sqrt{a}}\right)} \frac{\left(\frac{a}{k} ; q^{2}\right)_{n-j}}{\left(q^{2} ; q^{2}\right)_{n-j}} \frac{\left(k ; q^{2}\right)_{n+j}}{\left(\frac{k^{2} q^{2}}{a} ; q^{2}\right)_{n+j}} \\
& \times\left(\frac{k}{a}\right)^{n-j} \beta_{j}\left(\sqrt{a}, \frac{k}{\sqrt{a}}, q\right) .
\end{aligned}
$$


Proof. Set $e=\sqrt{a}, c=k / \sqrt{a}, g_{n}=q^{-n}\left(1+\sqrt{a} q^{n}\right) /(1+\sqrt{a})$. To get the inner sum over $r$ in (3.3) in closed form, we use the following result of Andrews and Berkovich [2] (we replace $\sqrt{q}$ with $q$ ):

$$
\begin{aligned}
{ }_{10} W_{9}\left(\sqrt{a} ; i q a^{1 / 4},-i q a^{1 / 4}\right. & \left., \sqrt{k} q^{n},-\sqrt{k} q^{n}, q^{-n},-q^{-n}, a / k ; q ; q\right) \\
& =\frac{\left(a q^{2}, a / k ; q^{2}\right)_{n}}{\left(k / a, k^{2} / a ; q^{2}\right)_{n}} \frac{(-k q / \sqrt{a} ; q)_{2 n}}{(\sqrt{a} ; q)_{2 n}}\left(\frac{k}{a q}\right)^{n},
\end{aligned}
$$

with $a$ replaced with $a q^{4 j}, k$ with $k q^{4 j}$ and $n$ with $n-j$. The result follows once again, after some $q$-product manipulations.

The next result also appears to be new.

Corollary 7. If $\left(\alpha_{n}(a, k, q), \beta_{n}(a, k, q)\right)$ satisfy (1.1), then so do $\left(\alpha_{n}^{\prime}(a, k, q)\right.$, $\left.\beta_{n}^{\prime}(a, k, q)\right)$, where

$$
\begin{aligned}
& \alpha_{n}^{\prime}\left(a, k, q^{2}\right)= \alpha_{n}\left(\sqrt{a}, \frac{k}{\sqrt{a}}, q\right) \\
& \beta_{n}^{\prime}\left(a, k, q^{2}\right)=\frac{\left(\frac{-k}{\sqrt{a}} ; q\right)_{2 n}}{(-q \sqrt{a} ; q)_{2 n}} \sum_{j=0}^{n} \frac{\left(1-\frac{k^{2} q^{4 j}}{a}\right)}{\left(1-\frac{k^{2}}{a}\right)} \frac{\left(\frac{a}{k} ; q^{2}\right)_{n-j}}{\left(q^{2} ; q^{2}\right)_{n-j}} \frac{\left(k ; q^{2}\right)_{n+j}}{\left(\frac{k^{2} q^{2}}{a} ; q^{2}\right)_{n+j}} \\
& \times\left(\frac{k q}{a}\right)^{n-j} \beta_{j}\left(\sqrt{a}, \frac{k}{\sqrt{a}}, q\right) .
\end{aligned}
$$

Proof. Set $e=\sqrt{a}, c=k / \sqrt{a}, g_{n}=1$. This time, to get the inner sum over $r$ in (3.3) in closed form, we use the following result of Warnaar [11]:

$$
\begin{aligned}
{ }_{8} W_{7}\left(a ; b, a q^{n} / \sqrt{b},-a q^{n} / \sqrt{b}\right. & \left., q^{-n},-q^{-n} ; q ; q^{2}\right) \\
& =\frac{(-a / b ; q)_{2 n}}{(-a q ; q)_{2 n}} \frac{\left(a^{2} q^{2}, b ; q^{2}\right)_{n}}{\left(1 / b, a^{2} q^{2} / b^{2} ; q^{2}\right)_{n}}\left(\frac{q}{b}\right)^{n},
\end{aligned}
$$

with $a$ replaced with $\sqrt{a} q^{2 j}, b$ with $a / k$ and $n$ with $n-j$. The result follows, as above, after some $q$-product manipulations.

Inserting the unit pair (4.1) in this chain gives the pair

$$
\begin{aligned}
& \alpha_{n}(a, k, q)=\frac{1-\sqrt{a} q^{n}}{1-\sqrt{a}} \frac{\left(\sqrt{a}, \frac{a}{k} ; \sqrt{q}\right)_{n}}{\left(\sqrt{q}, k \sqrt{\frac{q}{a}} ; \sqrt{q}\right)_{n}}\left(\frac{k}{a}\right)^{n}, \\
& \beta_{n}(a, k, q)=\frac{\left(-\frac{k}{\sqrt{a}} ; \sqrt{q}\right)_{2 n}}{(-\sqrt{a q} ; \sqrt{q})_{2 n}} \frac{\left(\frac{a}{k}, k ; q\right)_{n}}{\left(\frac{k^{2} q}{a}, q ; q\right)_{n}}\left(\frac{k \sqrt{q}}{a}\right)^{n},
\end{aligned}
$$

which is somewhat reminiscent of Bressoud's pair at (4.10).

Motivated by Warnaar's Theorem 2.6 in [11, we also have the following general WP-Bailey chain. 
Theorem 4. Suppose that $\left(\alpha_{n}(a, k, q), \beta_{n}(a, k, q)\right)$ satisfy (1.1), that $g_{n}$ is an arbitrary sequence of functions and that $e$ and $c$ are arbitrary constants. Then $\left(\alpha_{n}^{\prime}(a, k, q), \beta_{n}^{\prime}(a, k, q)\right)$ also satisfy (1.1), where

$$
\begin{aligned}
& \text { (3.10) } \quad \alpha_{2 n}^{\prime}(a, k, q)=g_{n} \alpha_{n}\left(e, c, q^{2}\right), \quad \alpha_{2 n+1}(a, k, q)=0, \\
& \beta_{n}^{\prime}(a, k, q)=\sum_{j=0}^{n} \beta_{j}\left(e, c, q^{2}\right) \frac{\left(1-c q^{4 j}\right)\left(\frac{k}{a} ; q\right)_{n-2 j}(k ; q)_{n+2 j}\left(e q^{2} ; q^{2}\right)_{2 j}}{(1-c)(q ; q)_{n-2 j}(a q ; q)_{n+2 j}\left(c q^{2} ; q^{2}\right)_{2 j}} \\
& \times \sum_{r=0}^{\lfloor n / 2\rfloor-j} \frac{\left(1-e q^{4 j+4 r}\right)\left(q^{-(n-2 j)}, k q^{n+2 j} ; q\right)_{2 r}\left(e / c, e q^{4 j} ; q^{2}\right)_{r} g_{r+j}}{\left(1-e q^{4 j}\right)\left(a q^{n+2 j+1}, a q^{1-(n-2 j)} / k ; q\right)_{2 r}\left(c q^{4 j+2}, q^{2} ; q^{2}\right)_{r}}\left(\frac{q^{2} c a^{2}}{k^{2} e}\right)^{r} .
\end{aligned}
$$

Once again, we omit the proof, since it is very similar to the proofs of the other theorems above.

Upon setting $g_{n}=1, e=a$ and $c=k^{2} / a$, and once again using (2.10), we obtain the WP-Bailey chain of Warnaar in Theorem 2.6 of [11.

Corollary 8 (Warnaar, [1]). If $\left(\alpha_{n}(a, k, q), \beta_{n}(a, k, q)\right)$ satisfy (1.1), then so do $\left(\alpha_{n}^{\prime}(a, k, q), \beta_{n}^{\prime}(a, k, q)\right)$, where

$$
\begin{aligned}
& \alpha_{2 n}^{\prime}(a, k, q)= \alpha_{n}\left(a, \frac{k^{2}}{a}, q^{2}\right), \quad \alpha_{2 n+1}^{\prime}(a, k, q)=0 \\
& \beta_{n}^{\prime}(a, k, q)=\frac{\left(\frac{k^{2} q}{a} ; q^{2}\right)_{n}}{\left(a q ; q^{2}\right)_{n}} \sum_{j=0}^{\lfloor n / 2\rfloor} \frac{\left(1-\frac{k^{2} q^{4 j}}{a}\right)}{\left(1-\frac{k^{2}}{a}\right)} \frac{\left(\frac{a}{k} ; q\right)_{n-2 j}}{(q ; q)_{n-2 j}} \frac{(k ; q)_{n+2 j}}{\left(\frac{k^{2} q}{a} ; q\right)_{n+2 j}} \\
& \times\left(\frac{-k}{a}\right)^{n-2 j} \beta_{j}\left(a, \frac{k^{2}}{a}, q^{2}\right) .
\end{aligned}
$$

\section{Transformation Formulae for Basic Hypergeometric Series}

We next describe a number of transformations of basic hypergeometric series which follow from the new WP-Bailey chains described in Corollaries 3. 4 and 7 .

Before proceeding, we remark that the ten or more WP-Bailey chains that presently exist potentially give rise to two hundred or more transformations between basic hypergeometric series, most of which have probably not been written down (by inserting the ten or more WP-Bailey pairs and their "duals" via Corollary 1 into the ten or more existing WP-Bailey chains, ignoring possible duplication (which is why we write "potentially")).

Inserting the unit WP-Bailey pair (see [2] for example, where this WPBailey pair, and others employed below, may be found),

$$
\begin{aligned}
& \alpha_{n}(a, k)=\frac{(q \sqrt{a},-q \sqrt{a}, a, a / k ; q)_{n}}{(\sqrt{a},-\sqrt{a}, q, k q ; q)_{n}}\left(\frac{k}{a}\right)^{n}, \\
& \beta_{n}(a, k)= \begin{cases}1 & n=0, \\
0, & n>1,\end{cases}
\end{aligned}
$$


into (2.15) leads (perhaps not surprisingly) to (2.13). Upon substituting Singh's WP-Bailey pair [9],

$$
\begin{aligned}
\alpha_{n}(a, k) & =\frac{\left(q \sqrt{a},-q \sqrt{a}, a, \rho_{1}, \rho_{2}, a^{2} q / k \rho_{1} \rho_{2} ; q\right)_{n}}{\left(\sqrt{a},-\sqrt{a}, q, a q / \rho_{1}, a q / \rho_{2}, k \rho_{1} \rho_{2} / a ; q\right)_{n}}\left(\frac{k}{a}\right)^{n}, \\
\beta_{n}(a, k) & =\frac{\left(k \rho_{1} / a, k \rho_{2} / a, k, a q / \rho_{1} \rho_{2} ; q\right)_{n}}{\left(a q / \rho_{1}, a q / \rho_{2}, k \rho_{1} \rho_{2} / a, q ; q\right)_{n}}
\end{aligned}
$$

into (2.15) we get the following transformation:

$$
\begin{aligned}
& { }_{12} \phi_{11}\left[\begin{array}{c}
a, q \sqrt{a},-q \sqrt{a}, a \sqrt{\frac{q}{k}},-a \sqrt{\frac{q}{k}}, \frac{a}{\sqrt{k}},-\frac{a}{\sqrt{k}}, \rho_{1}, \rho_{2}, \frac{k q^{2}}{\rho_{1} \rho_{2}}, k q^{n}, q^{-n} \\
\left.\sqrt{a},-\sqrt{a}, \sqrt{k q},-\sqrt{k q}, q \sqrt{k},-q \sqrt{k}, \frac{a q}{\rho_{1}}, \frac{a q}{\rho_{2}}, \frac{a \rho_{1} \rho_{2}}{k q}, \frac{a q^{1-n}}{k}, a q^{n+1} ; q, q\right]
\end{array}\right] \\
& =\frac{1-k}{1-k q^{2 n}} \frac{\left(a q, \frac{k^{2} q}{a^{2}} ; q\right)_{n}}{\left(k, \frac{k}{a} ; q\right)_{n}}{ }_{7} \phi_{6}\left[\begin{array}{c}
\frac{a^{2}}{k q}, q \sqrt{\frac{a^{2}}{k q}},-q \sqrt{\frac{a^{2}}{k q}}, \frac{a \rho_{1}}{k q}, \frac{a \rho_{2}}{k q}, \frac{a q}{\rho_{1} \rho_{2}}, q^{-n} \\
\sqrt{\frac{a^{2}}{k q}},-\sqrt{\frac{a^{2}}{k q}}, \frac{a q}{\rho_{1}}, \frac{a q}{\rho_{2}}, \frac{a \rho_{1} \rho_{2}}{k q}, \frac{a^{2} q^{-n}}{k^{2}}
\end{array} ; q, q\right] .
\end{aligned}
$$

It is easy to recognize this as Bailey's ${ }_{12} W_{11} \rightarrow{ }_{7} \phi_{6}$ transformation (see [6, III.27]). Note that (2.13) follows immediately from (4.3), upon setting $\rho_{1}=a q / \rho_{2}$. Inserting the pair

$$
\begin{aligned}
& \alpha_{n}(a, k)=\frac{(q \sqrt{a},-q \sqrt{a}, a, k / a q ; q)_{n}}{\left(\sqrt{a},-\sqrt{a}, q, a^{2} q^{2} / k ; q\right)_{n}} \frac{\left(q a^{2} / k ; q\right)_{2 n}}{(k ; q)_{2 n}}\left(\frac{k}{a}\right)^{n}, \\
& \beta_{n}(a, k)=\frac{\left(k^{2} / q a^{2} ; q\right)_{n}}{(q ; q)_{n}},
\end{aligned}
$$

into (2.15) leads to

$$
\begin{gathered}
{ }_{10} \phi_{9}\left[\begin{array}{c}
\left.a, q \sqrt{a},-q \sqrt{a}, a \sqrt{\frac{q}{k}},-a \sqrt{\frac{q}{k}}, q \sqrt{k q},-q \sqrt{k q}, \frac{a}{k q^{2}}, k q^{n}, q^{-n} ; q, q\right] \\
\sqrt{a},-\sqrt{a}, \sqrt{k q},-\sqrt{k q}, \frac{a}{\sqrt{k q}}, \frac{-a}{\sqrt{k q}}, k q^{3}, \frac{a q^{1-n}}{k}, a q^{n+1}
\end{array}\right] \\
=\frac{1-k}{1-k q^{2 n}} \frac{\left(a q, \frac{k^{2} q}{a^{2}} ; q\right)_{n}}{\left(k, \frac{k}{a} ; q\right)_{n}} \phi_{3}\left[\begin{array}{c}
\left.q \sqrt{\frac{a^{2}}{k q}},-q \sqrt{\frac{a^{2}}{k q}}, \frac{a^{2}}{k^{2} q^{3}}, q^{-n} ; q, q\right] \\
\sqrt{\frac{a^{2}}{k q}},-\sqrt{\frac{a^{2}}{k q}}, \frac{a^{2} q^{-n}}{k^{2}}
\end{array}\right] .
\end{gathered}
$$

Similarly, inserting the pair

$$
\begin{aligned}
& \left.\alpha_{n}(a, k)=\frac{\left(q \sqrt{a},-q \sqrt{a}, a, a \sqrt{\frac{q}{k}},-a \sqrt{\frac{q}{k}}, \frac{a}{\sqrt{k}},-\frac{a q}{\sqrt{k}}, \frac{k}{a} ; q\right)_{n}}{\left(\sqrt{a},-\sqrt{a}, q, \sqrt{k q},-\sqrt{k q}, q \sqrt{k},-\sqrt{k}, \frac{q a^{2}}{k} ; q\right)_{n}}\right)^{n}, \\
& \beta_{n}(a, k)=\frac{\left(\sqrt{k}, \frac{k^{2}}{a^{2}} ; q\right)_{n}}{(q \sqrt{k}, q ; q)_{n}}
\end{aligned}
$$


into (2.15) leads to

$$
\begin{aligned}
& { }_{8} \phi_{7}\left[\begin{array}{c}
a, q \sqrt{a},-q \sqrt{a},-a \sqrt{\frac{q}{k}},-q \sqrt{k q}, \frac{a}{k q}, k q^{n}, q^{-n} \\
\sqrt{a},-\sqrt{a},-\sqrt{k q}, \frac{-a}{\sqrt{k q}}, k q^{2}, \frac{a q^{1-n}}{k}, a q^{n+1}
\end{array} ; q, q\right] \\
& =\frac{1-k}{1-k q^{2 n}} \frac{\left(a q, \frac{k^{2} q}{a^{2}} ; q\right)_{n}}{\left(k, \frac{k}{a} ; q\right)_{n}}{ }_{5} \phi_{4}\left[\begin{array}{c}
q \sqrt{\frac{a^{2}}{k q}},-q \sqrt{\frac{a^{2}}{k q}}, \frac{a}{\sqrt{k q}}, \frac{a^{2}}{k^{2} q^{2}}, q^{-n} \\
\sqrt{\frac{a^{2}}{k q}},-\sqrt{\frac{a^{2}}{k q}}, a \sqrt{\frac{q}{k}}, \frac{a^{2} q^{-n}}{k^{2}}
\end{array} ; q, q\right] .
\end{aligned}
$$

Substituting the pair

$$
\begin{aligned}
& \alpha_{n}(a, k)= \begin{cases}0, & \text { if } n \text { is odd }, \\
\frac{\left(q^{2} \sqrt{a},-q^{2} \sqrt{a}, a, a^{2} / k^{2} ; q^{2}\right)_{n / 2}}{\left(\sqrt{a},-\sqrt{a}, q^{2}, q^{2} k^{2} / a ; q^{2}\right)_{n / 2}}\left(\frac{k}{a}\right)^{n}, & \text { if } n \text { is even, }\end{cases} \\
& \beta_{n}(a, k)=\frac{(k, k \sqrt{q / a},-k \sqrt{q / a}, a / k ; q)_{n}}{\left(\sqrt{a q},-\sqrt{a q}, q k^{2} / a, q ; q\right)_{n}}\left(\frac{-k}{a}\right)^{n},
\end{aligned}
$$

into (2.15) leads to

$$
\begin{aligned}
& { }_{16} W_{15}\left(a ; \frac{a}{\sqrt{k}}, \frac{-a}{\sqrt{k}}, a \sqrt{\frac{q}{k}},-a \sqrt{\frac{q}{k}}, \frac{a q}{\sqrt{k}}, \frac{-a q}{\sqrt{k}}, a q \sqrt{\frac{q}{k}},-a q \sqrt{\frac{q}{k}}, \frac{k^{2} q^{2}}{a^{2}}, k q^{n}, k q^{n+1}, q^{-n}, q^{1-n} ; q^{2}, q^{2}\right) \\
& =\frac{1-k}{1-k q^{2 n}} \frac{\left(a q, \frac{k^{2} q}{a^{2}} ; q\right)_{n}}{\left(k, \frac{k}{a} ; q\right)_{n}} \phi_{6}\left[\begin{array}{l}
\frac{a q}{\sqrt{k q}},-\frac{a q}{\sqrt{k q}}, \frac{a^{2}}{k q}, \frac{a}{k} \sqrt{\frac{a}{q}},-\frac{a}{k} \sqrt{\frac{a}{q}}, \frac{k q}{a}, q^{-n} \\
\left.\frac{a}{\sqrt{k q}},-\frac{a}{\sqrt{k q}}, \sqrt{a q},-\sqrt{a q}, \frac{a^{3}}{k^{2} q}, \frac{a^{2} q^{-n}}{k^{2}} ; q, q\right],
\end{array}\right]
\end{aligned}
$$

a result reminiscent of (3.14) in [2].

We next turn to two WP-Bailey pairs found by Bressoud [3]. Inserting the pair

$$
\begin{aligned}
& \alpha_{n}(a, k)=\frac{1-\sqrt{a} q^{n}}{1-\sqrt{a}} \frac{\left(\sqrt{a}, \frac{a \sqrt{q}}{k} ; \sqrt{q}\right)_{n}}{\left(\sqrt{q}, \frac{k}{\sqrt{a}} ; \sqrt{q}\right)_{n}}\left(\frac{k}{a \sqrt{q}}\right)^{n}, \\
& \beta_{n}(a, k)=\frac{\left(k, \frac{a q}{k} ; q\right)_{n}}{\left(q, \frac{k^{2}}{a} ; q\right)_{n}} \frac{\left(\frac{-k}{\sqrt{a}} ; \sqrt{q}\right)_{2 n}}{(-\sqrt{a q} ; \sqrt{q})_{2 n}}\left(\frac{k}{a \sqrt{q}}\right)^{n},
\end{aligned}
$$

into (2.15), and then replacing $\sqrt{a}$ with $a, \sqrt{k}$ with $k$ and $\sqrt{q}$ with $q$, gives

$$
\sum_{j=0}^{n} \frac{\left(q \sqrt{a},-q \sqrt{a}, a, \frac{k^{2} q^{3}}{a^{2}} ; q\right)_{j}}{\left(\sqrt{a},-\sqrt{a}, \frac{a^{3}}{q^{2} k^{2}}, q ; q\right)_{j}} \frac{\left(\frac{a^{2}}{k},-\frac{a^{2}}{k}, \frac{a^{2} q}{k},-\frac{a^{2} q}{k}, k^{2} q^{2 n}, q^{-2 n} ; q^{2}\right)_{j} q^{j}}{\left(k q,-k q, k q^{2},-k q^{2}, \frac{a^{2} q^{2-2 n}}{k^{2}}, a^{2} q^{2+n} ; q^{2}\right)_{j}}
$$




$$
\begin{gathered}
=\frac{1-k^{2}}{1-k^{2} q^{4 n}} \frac{\left(a^{2} q^{2}, \frac{k^{4} q^{2}}{a^{4}} ; q^{2}\right)_{n}}{\left(k^{2}, \frac{k^{2}}{a^{2}} ; q^{2}\right)_{n}} \\
\times{ }_{7} \phi_{6}\left[\begin{array}{c}
\frac{a^{2} q^{2}}{k q},-\frac{a^{2} q^{2}}{k q}, \frac{a^{4}}{k^{2} q^{2}}, \frac{-a^{3}}{k^{2} q^{2}}, \frac{-a^{3}}{k^{2} q}, \frac{k^{2} q^{4}}{a^{2}}, q^{-2 n} \\
\frac{a^{2}}{k q},-\frac{a^{2}}{k q},-a q,-a q^{2}, \frac{a^{6}}{k^{4} q^{4}}, \frac{a^{4} q^{-2 n}}{k^{4}}
\end{array} ; q^{2}, \frac{a^{2}}{k^{2} q}\right],
\end{gathered}
$$

an identity similar to (4.10) in [2]. In a similar manner, inserting Bressoud's WP-Bailey pair

$$
\begin{aligned}
& \alpha_{n}(a, k)=\frac{1-a q^{2 n}}{1-a} \frac{\left(\sqrt{a}, \frac{a}{k} ; \sqrt{q}\right)_{n}}{\left(\sqrt{q}, k \sqrt{\frac{q}{a}} ; \sqrt{q}\right)_{n}}\left(\frac{k}{a \sqrt{q}}\right)^{n}, \\
& \beta_{n}(a, k)=\frac{\left(k, \frac{a}{k},-k \sqrt{\frac{q}{a}},-\frac{k q}{\sqrt{a}} ; q\right)_{n}}{\left(q, \frac{q k^{2}}{a},-\sqrt{a},-\sqrt{a q} ; q\right)_{n}}\left(\frac{k}{a \sqrt{q}}\right)^{n},
\end{aligned}
$$

into (2.15), and likewise replacing $\sqrt{a}$ with $a, \sqrt{k}$ with $k$ and $\sqrt{q}$ with $q$, gives

$$
\begin{aligned}
& \sum_{j=0}^{n} \frac{\left(a, \frac{k^{2} q^{2}}{a^{2}} ; q\right)_{j}}{\left(\frac{a^{3}}{q k^{2}}, q ; q\right)_{j}} \frac{\left(a q^{2},-a q^{2}, \frac{a^{2}}{k},-\frac{a^{2}}{k}, \frac{a^{2} q}{k},-\frac{a^{2} q}{k}, k^{2} q^{2 n}, q^{-2 n} ; q^{2}\right)_{j} q^{j}}{\left(a,-a, k q,-k q, k q^{2},-k q^{2}, \frac{a^{2} q^{2-2 n}}{k^{2}}, a^{2} q^{2+n} ; q^{2}\right)_{j}} \\
& =\frac{1-k^{2}}{1-k^{2} q^{4 n}} \frac{\left(a^{2} q^{2}, \frac{k^{4} q^{2}}{a^{4}} ; q^{2}\right)_{n}}{\left(k^{2}, \frac{k^{2}}{a^{2}} ; q^{2}\right)_{n}} \\
& \times{ }_{7} \phi_{6}\left[\begin{array}{c}
\frac{a^{2} q}{k},-\frac{a^{2} q}{k}, \frac{a^{4}}{k^{2} q^{2}}, \frac{-a^{3}}{k^{2} q}, \frac{-a^{3}}{k^{2}}, \frac{k^{2} q^{2}}{a^{2}}, q^{-2 n} \\
\frac{a^{2}}{k q},-\frac{a^{2}}{k q},-a,-a q, \frac{a^{6}}{k^{4} q^{2}}, \frac{a^{4} q^{-2 n}}{k^{4}}
\end{array} ; q^{2}, \frac{a^{2}}{k^{2} q}\right] .
\end{aligned}
$$

Finally, we apply the corollary to two WP-Bailey pairs found by the present authors in [8]:

$$
\begin{gathered}
\alpha_{n}^{(1)}(a, k)=\frac{\left(q a^{2} / k^{2} ; q\right)_{n}}{(q, q)_{n}}\left(\frac{k}{a}\right)^{n}, \\
\beta_{n}^{(1)}(a, k)=\frac{(q a / k, k ; q)_{n}}{\left(k^{2} / a, q, q\right)_{n}} \frac{\left(k^{2} / a ; q\right)_{2 n}}{(a q, q)_{2 n}} . \\
\alpha_{n}^{(2)}(a, k)=\frac{(a, q \sqrt{a},-q \sqrt{a}, k / a, a \sqrt{q / k},-a \sqrt{q / k} ; q)_{n}}{\left(\sqrt{a},-\sqrt{a}, q a^{2} / k, \sqrt{q k},-\sqrt{q k}, q ; q\right)_{n}}(-1)^{n}, \\
\beta_{n}^{(2)}(a, k)= \begin{cases}\frac{\left(k, k^{2} / a^{2} ; q^{2}\right)_{n / 2}}{\left(q^{2}, q^{2} a^{2} / k ; q^{2}\right)_{n / 2}}, & n \text { even, } \\
0, & n \text { odd. }\end{cases}
\end{gathered}
$$


Note that the second pair may also be derived by applying Corollary 1 to the pair at (4.8). These WP-Bailey pairs inserted in (2.15) lead, respectively to the transformations

$$
\begin{aligned}
& { }_{7} \phi_{6}\left[\begin{array}{c}
\frac{a}{\sqrt{k}}, \frac{-a}{\sqrt{k}}, a \sqrt{\frac{q}{k}},-a \sqrt{\frac{q}{k}}, \frac{k^{2} q^{3}}{a^{2}}, k q^{n}, q^{-n} \\
\sqrt{k q},-\sqrt{k q}, q \sqrt{k},-q \sqrt{k}, \frac{a q^{1-n}}{k}, a q^{n+1}
\end{array}\right] \\
& =\frac{1-k}{1-k q^{2 n}} \frac{\left(a q, \frac{k^{2} q}{a^{2}} ; q\right)_{n}}{\left(k, \frac{k}{a} ; q\right)_{n}}
\end{aligned}
$$

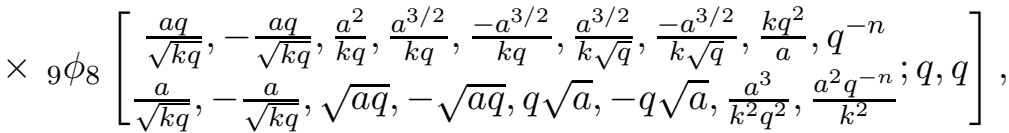

and

$$
\begin{aligned}
{ }_{8} \phi_{7}\left[\begin{array}{l}
a, q \sqrt{a},-q \sqrt{a}, a \sqrt{\frac{q}{k}},-a \sqrt{\frac{q}{k}}, \frac{a}{k q}, k q^{n}, q^{-n} \\
\left.\sqrt{a},-\sqrt{a}, \sqrt{k q},-\sqrt{k q}, q^{2} k, \frac{a q^{1-n}}{k}, a q^{n+1} ; q,-\frac{k q^{2}}{a}\right]
\end{array}\right. \\
=\frac{1-k}{1-k q^{2 n}} \frac{\left(a q, \frac{k^{2} q}{a^{2}} ; q\right)_{n}}{\left(k, \frac{k}{a} ; q\right)_{n}} 6 \phi_{5}\left[\begin{array}{l}
\frac{a q^{2}}{\sqrt{k q}},-\frac{a q^{2}}{\sqrt{k q}}, \frac{a^{2}}{k q}, \frac{a^{2}}{k^{2} q^{2}}, q^{-n}, q^{1-n} \\
\frac{a}{\sqrt{k q}},-\frac{a}{\sqrt{k q}}, q^{3} k, \frac{a^{2} q^{-n}}{k^{2}}, \frac{a^{2} q^{1-n}}{k^{2}} ; q^{2}, q^{2}
\end{array}\right] .
\end{aligned}
$$

Likewise, inserting the WP-Bailey pairs at (4.2), (4.4), (4.6), (4.8), (4.10), (4.12), (4.14) and (4.15), respectively, into the WP-Bailey chain at (3.2) leads, respectively, to the following transformations:

$$
\begin{aligned}
& { }_{10} W_{9}\left(\frac{a k}{q} ;-a,-a q, \frac{k \rho_{1}}{a q}, \frac{k \rho_{2}}{a q}, \frac{a^{2} q^{2}}{\rho_{1} \rho_{2}}, k^{2} q^{2 n}, q^{-2 n} ; q^{2}, \frac{q^{2} a}{k}\right) \\
& =\frac{(k / a,-q ; q)_{n}}{(-k, a q ; q)_{n}} \frac{\left(a k q ; q^{2}\right)_{n}}{\left(k q / a ; q^{2}\right)_{n}} \\
& \times \sum_{j=0}^{n} \frac{\left(k q^{n}, q^{-n} ; q\right)_{j}}{\left(\frac{a q^{1-n}}{k}, a q^{1+n} ; q\right)_{j}} \frac{\left(a^{2}, a q^{2}, \rho_{1}, \rho_{2}, \frac{a^{3} q^{3}}{k \rho_{1} \rho_{2}} ; q^{2}\right)_{j}}{\left(a, \frac{a^{2} q^{2}}{\rho_{1}}, \frac{a^{2} q^{2}}{\rho_{2}}, \frac{k \rho_{1} \rho_{2}}{a q}, q^{2} ; q^{2}\right)_{j}} q^{j} ; \\
& { }_{7} \phi_{6}\left[\begin{array}{c}
q^{2} \sqrt{\frac{a k}{q}},-q^{2} \sqrt{\frac{a k}{q}},-a,-a q, \frac{k^{2}}{a^{2} q^{4}}, k^{2} q^{2 n}, q^{-2 n} \\
\sqrt{\frac{a k}{q}},-\sqrt{\frac{a k}{q}},-k,-k q, a k q^{2 n+1}, \frac{a q^{1-2 n}}{k}
\end{array} ; q^{2}, \frac{q^{2} a}{k}\right] \\
& =\frac{(k / a,-q ; q)_{n}}{(-k, a q ; q)_{n}} \frac{\left(a k q ; q^{2}\right)_{n}}{\left(k q / a ; q^{2}\right)_{n}} \\
& \times \sum_{j=0}^{n} \frac{\left(k q^{n}, q^{-n} ; q\right)_{j}}{\left(\frac{a q^{1-n}}{k}, a q^{1+n} ; q\right)_{j}} \frac{\left(a^{2}, a q^{2}, \frac{k}{a q^{3}} ; q^{2}\right)_{j}}{\left(a, \frac{a^{3} q^{5}}{k}, q^{2} ; q^{2}\right)_{j}} \frac{\left(\frac{q^{3} a^{3}}{k}, \frac{q^{5} a^{3}}{k} ; q^{4}\right)_{j}}{\left(\frac{a k}{q}, a k q ; q^{4}\right)_{j}} q^{j}
\end{aligned}
$$




$$
\begin{aligned}
& { }_{6} \phi_{5}\left[\begin{array}{c}
-q^{2} \sqrt{\frac{a k}{q}},-a,-a q, \frac{k^{2}}{a^{2} q^{2}}, k^{2} q^{2 n}, q^{-2 n} \\
-\sqrt{\frac{a k}{q}},-k,-k q, a k q^{2 n+1}, \frac{a q^{1-2 n}}{k}
\end{array} ; q^{2}, \frac{q^{2} a}{k}\right] \\
& =\frac{(k / a,-q ; q)_{n}}{(-k, a q ; q)_{n}} \frac{\left(a k q ; q^{2}\right)_{n}}{\left(k q / a ; q^{2}\right)_{n}} \\
& \times \sum_{j=0}^{n} \frac{\left(k q^{n}, q^{-n} ; q\right)_{j}}{\left(\frac{a q^{1-n}}{k}, a q^{1+n} ; q\right)_{j}} \frac{\left(a^{2}, a q^{2}, \frac{k}{a q}, \sqrt{\frac{a^{3} q}{k}},-\sqrt{\frac{a^{3} q^{5}}{k}} ; q^{2}\right)_{j}}{\left(a, \frac{a^{3} q^{3}}{k}, \sqrt{a k q^{3}},-\sqrt{\frac{a k}{q}}, q^{2} ; q^{2}\right)_{j}} \frac{\left(\frac{q^{3} a^{3}}{k} ; q^{4}\right)_{j}}{\left(a k q ; q^{4}\right)_{j}} q^{j} ;
\end{aligned}
$$

$$
\begin{aligned}
{ }_{8} W_{7}\left(\frac{a k}{q} ;-a, k,\right. & \left.\frac{a q}{k}, k^{2} q^{2 n}, q^{-2 n} ; q^{2},-q\right)=\frac{(k / a,-q ; q)_{n}}{(-k, a q ; q)_{n}} \frac{\left(a k q ; q^{2}\right)_{n}}{\left(k q / a ; q^{2}\right)_{n}} \\
& \times{ }_{10} W_{9}\left(a ;-a, \frac{a q}{k},-\frac{a q}{k}, k q^{n}, k q^{1+n}, q^{1-n}, q^{-n} ; q^{2}, q^{2}\right)
\end{aligned}
$$

$$
\begin{array}{r}
{ }_{8} W_{7}\left(\frac{a k}{q} ;-a,-\frac{k}{q}, \frac{a q^{3}}{k}, k^{2} q^{2 n}, q^{-2 n} ; q^{2}, 1\right)=\frac{(k / a,-q ; q)_{n}}{(-k, a q ; q)_{n}} \frac{\left(a k q ; q^{2}\right)_{n}}{\left(k q / a ; q^{2}\right)_{n}} \\
\times{ }_{8} W_{7}\left(a ; i \sqrt{a},-i \sqrt{a}, \frac{a q^{2}}{k}, k q^{n}, q^{-n} ; q, 1\right) ;
\end{array}
$$

$$
\begin{aligned}
{ }_{6} W_{5}\left(\frac{a k}{q} ; \frac{a q}{k}, k^{2} q^{2 n}, q^{-2 n} ; q^{2}, 1\right)= & \frac{(k / a,-q ; q)_{n}}{(-k, a q ; q)_{n}} \frac{\left(a k q ; q^{2}\right)_{n}}{\left(k q / a ; q^{2}\right)_{n}} \\
& \times_{6} W_{5}\left(a ; \frac{a q}{k}, k q^{n}, q^{-n} ; q, 1\right)
\end{aligned}
$$

$$
\begin{aligned}
& { }_{10} W_{9}\left(\frac{a k}{q} ;-a, k, \frac{k}{q},-\frac{k}{q}, \frac{a q^{3}}{k}, k^{2} q^{2 n}, q^{-2 n} ; q^{2}, \frac{q^{2} a}{k}\right)= \\
& \frac{(k / a,-q ; q)_{n}}{(-k, a q ; q)_{n}} \frac{\left(a k q ; q^{2}\right)_{n}}{\left(k q / a ; q^{2}\right)_{n}} \sum_{j=0}^{n} \frac{(1+a)\left(a q^{2} / k,-a q^{2} / k, k q^{n}, q^{-n} ; q\right)_{j}}{\left(1+a q^{2 j}\right)\left(a q^{1-n} / k, a q^{1+n},-q, q ; q\right)_{j}} q^{j}
\end{aligned}
$$

$$
\begin{aligned}
{ }_{12} W_{11} & \left(\frac{a k}{q} ;-a,-a q,-a q^{2},-a q^{3}, \frac{k^{2}}{a^{2} q^{2}}, k^{2} q^{2 n}, k^{2} q^{2+2 n}, q^{2-2 n}, q^{-2 n} ; q^{4}, \frac{q^{4} a^{2}}{k^{2}}\right) \\
= & \frac{(k / a,-q ; q)_{n}}{(-k, a q ; q)_{n}} \frac{\left(a k q ; q^{2}\right)_{n}}{\left(k q / a ; q^{2}\right)_{n}} \\
& \times \sum_{j=0}^{n} \frac{\left(k q^{n}, q^{-n} ; q\right)_{j}}{\left(\frac{a q^{1-n}}{k}, a q^{1+n} ; q\right)_{j}} \frac{\left(a^{2}, a q^{2}, \frac{k}{a q} ; q^{2}\right)_{j}}{\left(a, \frac{a^{3} q^{3}}{k}, q^{2} ; q^{2}\right)_{j}} \frac{\left(\frac{a^{3} q^{3}}{k} ; q^{4}\right)_{j}}{\left(a k q ; q^{4}\right)_{j}}\left(-\frac{q^{2} a}{k}\right)^{j} .
\end{aligned}
$$


In a similar manner, inserting the WP-Bailey pairs at (4.2), (4.4), (4.6), (4.8), (4.10), (4.12), (4.14) and (4.15), respectively, into the WP-Bailey chain at (3.7) leads, respectively, to the following transformations:

$$
\begin{aligned}
& { }_{10} \phi_{9}\left[\begin{array}{l}
a, q \sqrt{a},-q \sqrt{a}, \rho_{1}, \rho_{2}, \frac{a^{3} q}{k \rho_{1} \rho_{2}}, \sqrt{k} q^{n},-\sqrt{k} q^{n}, q^{-n},-q^{-n} \\
\sqrt{a},-\sqrt{a}, \frac{a q}{\rho_{1}}, \frac{a q}{\rho_{2}}, \frac{k \rho_{1} \rho_{2}}{a^{2}}, \frac{a q^{1-n}}{\sqrt{k}},-\frac{a q^{1-n}}{\sqrt{k}}, a q^{n+1},-a q^{2}
\end{array}\right] \\
& =\frac{\left(a^{2} q^{2}, \frac{a^{2}}{k} ; q^{2}\right)_{n}\left(\frac{-k}{a} ; q\right)_{2 n}}{\left(\frac{k}{a^{2}}, \frac{k^{2} q^{2}}{a^{2}} ; q^{2}\right)_{n}(-q a ; q)_{2 n}}\left(\frac{k q}{a^{2}}\right)^{n}
\end{aligned}
$$

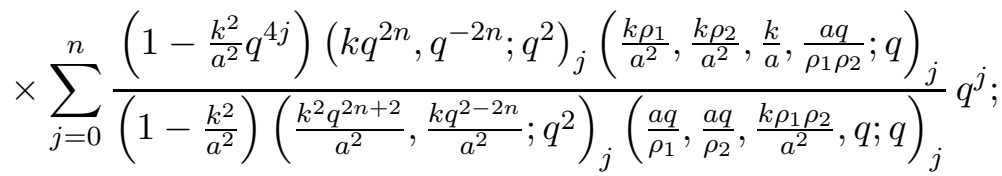

$$
\begin{aligned}
& \sum_{j=0}^{n} \frac{\left(a, q \sqrt{a},-q \sqrt{a}, \frac{k}{a^{2} q} ; q\right)_{j}\left(q^{-2 n}, k q^{2 n} ; q^{2}\right)_{j}\left(\frac{q a^{3}}{k} ; q\right)_{2 j}}{\left(\sqrt{a},-\sqrt{a}, q, \frac{a^{3} q^{2}}{k} ; q\right)_{j}\left(\frac{a^{2} q^{2-2 n}}{k}, a^{2} q^{2 n+2} ; q^{2}\right)_{j}\left(\frac{k}{a} ; q\right)_{2 j}} q^{2 j} \\
& =\frac{\left(a^{2} q^{2}, \frac{a^{2}}{k} ; q^{2}\right)_{n}\left(\frac{-k}{a} ; q\right)_{2 n}}{\left(\frac{k}{a^{2}}, \frac{k^{2} q^{2}}{a^{2}} ; q^{2}\right)_{n}(-q a ; q)_{2 n}}\left(\frac{k q}{a^{2}}\right)^{n} \\
& \times \sum_{j=0}^{n} \frac{\left(1-\frac{k^{2}}{a^{2}} q^{4 j}\right)\left(k q^{2 n}, q^{-2 n} ; q^{2}\right)_{j}\left(\frac{k^{2}}{q a^{4}} ; q\right)_{j}}{\left(1-\frac{k^{2}}{a^{2}}\right)\left(\frac{k^{2} q^{2 n+2}}{a^{2}}, \frac{k q^{2-2 n}}{a^{2}} ; q^{2}\right)_{j}(q ; q)_{j}} q^{j} ;
\end{aligned}
$$

$$
\begin{gathered}
{ }_{12} \phi_{11}\left[\begin{array}{c}
a, q \sqrt{a},-q \sqrt{a}, a \sqrt{\frac{q a}{k}},-a \sqrt{\frac{q a}{k}}, a \sqrt{\frac{a}{k}},-a q \sqrt{\frac{a}{k}}, \frac{k}{a^{2}}, q^{-n},-q^{-n}, \sqrt{k} q^{n},-\sqrt{k} q^{n} \\
\sqrt{a},-\sqrt{a}, \sqrt{\frac{k q}{a}},-\sqrt{\frac{k q}{a}}, q \sqrt{\frac{k}{a}},-\sqrt{\frac{k}{a}}, \frac{q a^{3}}{k}, \frac{a q^{1-n}}{\sqrt{k}},-\frac{a q^{1-n}}{\sqrt{k}}, a q^{n+1},-a q^{n+1}
\end{array}\right] \\
=\frac{\left(a^{2} q^{2}, \frac{a^{2}}{k} ; q^{2}\right)_{n}\left(\frac{-k}{a} ; q\right)_{2 n}\left(\frac{k q}{a^{2}}\right)^{n}}{\left(\frac{k}{a^{2}}, \frac{k^{2} q^{2}}{a^{2}} ; q^{2}\right)_{n}(-q a ; q)_{2 n}} \\
\times \sum_{j=0}^{n} \frac{\left(1-\frac{k^{2}}{a^{2}} q^{4 j}\right)\left(k q^{2 n}, q^{-2 n} ; q^{2}\right)_{j}}{\left.\left(1-\frac{k^{2}}{a^{2}}\right)\left(\frac{k^{2} q^{2 n+2}}{a^{2}}, \frac{k q^{2-2 n}}{a^{2}} ; q^{2}\right)_{j}\left(q \sqrt{\frac{k}{a}}, \frac{k^{2}}{a^{4}} ; q\right)_{j} ; q\right)_{j}} q_{j}^{j} ; \\
\sum_{j=0}^{n} \frac{\left(q^{-4 n}, k q^{4 n} ; q^{2}\right)_{2 j}\left(a, q^{2} \sqrt{a},-q^{2} \sqrt{a}, \frac{a^{4}}{k^{2}} ; q^{2}\right)_{j} q^{4 j}}{\left(\frac{a^{2} q^{2-4 n}}{k}, a^{2} q^{4 n+2} ; q^{2}\right)_{2 j}\left(q^{2}, \sqrt{a},-\sqrt{a}, \frac{q^{2} k^{2}}{a^{3}} ; q^{2}\right)_{j}}
\end{gathered}
$$




$$
\begin{aligned}
& =\frac{\left(a^{2} q^{2}, \frac{a^{2}}{k} ; q^{2}\right)_{2 n}\left(\frac{-k}{a} ; q\right)_{4 n}}{\left(\frac{k}{a^{2}}, \frac{k^{2} q^{2}}{a^{2}} ; q^{2}\right)_{2 n}(-q a ; q)_{4 n}}\left(\frac{k q}{a^{2}}\right)^{2 n} \\
& \left.\times \sum_{j=0}^{2 n} \frac{\left(1-\frac{k^{2}}{a^{2}} q^{4 j}\right)\left(k q^{4 n}, q^{-4 n} ; q^{2}\right)_{j}\left(\frac{k}{a}, \frac{k \sqrt{a q}}{a^{2}},-\frac{k \sqrt{a q}}{a^{2}}, \frac{a^{2}}{k} ; q\right)_{j}}{\left(1-\frac{k^{2}}{a^{2}}\right)\left(\frac{k^{2} q^{4 n+2}}{a^{2}}, \frac{k q^{2-4 n}}{a^{2}} ; q^{2}\right)_{j}\left(\sqrt{a q},-\sqrt{a q}, \frac{q k^{2}}{a^{3}}, q ; q\right)_{j}}\right)^{j} ; \\
& \sum_{j=0}^{n} \frac{\left(1-\sqrt{a} q^{2 n}\right)\left(q^{-4 n}, k q^{4 n} ; q^{4}\right)_{j}\left(\sqrt{a}, \frac{a^{2} q}{k} ; q\right)_{j}}{(1-\sqrt{a})\left(\frac{a^{2} q^{4-4 n}}{k}, a^{2} q^{4 n+4} ; q^{4}\right)_{j}\left(q, \frac{k \sqrt{a}}{a^{2}} ; q\right)_{j}} q^{3 j} \\
& =\frac{\left(a^{2} q^{4}, \frac{a^{2}}{k} ; q^{4}\right)_{n}\left(\frac{-k}{a} ; q^{2}\right)_{2 n}}{\left(\frac{k}{a^{2}}, \frac{k^{2} q^{4}}{a^{2}} ; q^{4}\right)_{n}\left(-q^{2} a ; q^{2}\right)_{2 n}}\left(\frac{k q^{2}}{a^{2}}\right)^{n} \\
& \left.\times \sum_{j=0}^{n} \frac{\left(1-\frac{k^{2}}{a^{2}} q^{8 j}\right)\left(k q^{4 n}, q^{-4 n} ; q^{4}\right)_{j}\left(\frac{k}{a}, \frac{a^{2} q^{2}}{k} ; q^{2}\right)_{j}\left(\frac{-k \sqrt{a}}{a^{2}} ; q\right)_{2 j}}{\left(1-\frac{k^{2}}{a^{2}}\right)\left(\frac{k^{2} q^{4 n+4}}{a^{2}}, \frac{k q^{4-4 n}}{a^{2}} ; q^{4}\right)_{j}\left(q^{2}, \frac{k^{2}}{a^{3}} ; q^{2}\right)_{j}(-q \sqrt{a} ; q)_{2 j}}\right)^{j} ; \\
& \sum_{j=0}^{n} \frac{\left(1-a q^{4 j}\right)\left(q^{-4 n}, k q^{4 n} ; q^{4}\right)_{j}\left(\sqrt{a}, \frac{a^{2}}{k} ; q\right)_{j}}{(1-a)\left(\frac{a^{2} q^{4-4 n}}{k}, a^{2} q^{4 n+4} ; q^{4}\right)_{j}\left(q, \frac{k q \sqrt{a}}{a^{2}} ; q\right)_{j}} q^{3 j} \\
& =\frac{\left(a^{2} q^{4}, \frac{a^{2}}{k} ; q^{4}\right)_{n}\left(\frac{-k}{a} ; q^{2}\right)_{2 n}}{\left(\frac{k}{a^{2}}, \frac{k^{2} q^{4}}{a^{2}} ; q^{4}\right)_{n}\left(-q^{2} a ; q^{2}\right)_{2 n}}\left(\frac{k q^{2}}{a^{2}}\right)^{n} \\
& \left.\times \sum_{j=0}^{n} \frac{\left(1-\frac{k^{2}}{a^{2}} q^{8 j}\right)\left(k q^{4 n}, q^{-4 n} ; q^{4}\right)_{j}\left(\frac{k}{a}, \frac{a^{2}}{k},-\frac{k q \sqrt{a}}{a^{2}},-\frac{k q^{2} \sqrt{a}}{a^{2}} ; q^{2}\right)_{j}}{\left(1-\frac{k^{2}}{a^{2}}\right)\left(\frac{k^{2} q^{4 n+4}}{a^{2}}, \frac{k q^{4-4 n}}{a^{2}} ; q^{4}\right)_{j}\left(q^{2}, \frac{q^{2} k^{2}}{a^{3}},-\sqrt{a},-q \sqrt{a} ; q^{2}\right)_{j}}\right)^{j} ;
\end{aligned}
$$

$$
\begin{aligned}
& { }_{5} \phi_{4}\left[\begin{array}{l}
\frac{q a^{4}}{k^{2}}, q^{-n},-q^{-n}, \sqrt{k} q^{n},-\sqrt{k} q^{n} \\
\frac{a q^{1-n}}{\sqrt{k}},-\frac{a q^{1-n}}{\sqrt{k}}, a q^{n+1},-a q^{n+1} ; q, q^{2}
\end{array}\right]=\frac{\left(a^{2} q^{2}, \frac{a^{2}}{k} ; q^{2}\right)_{n}\left(\frac{-k}{a} ; q\right)_{2 n}}{\left(\frac{k}{a^{2}}, \frac{k^{2} q^{2}}{a^{2}} ; q^{2}\right)_{n}(-q a ; q)_{2 n}} \\
& \times\left(\frac{k q}{a^{2}}\right)^{n} \sum_{j=0}^{n} \frac{\left(1-\frac{k^{2}}{a^{2}} q^{4 j}\right)\left(k q^{2 n}, q^{-2 n} ; q^{2}\right)_{j}\left(\frac{q a^{2}}{k}, \frac{k}{a} ; q\right)_{j}\left(\frac{k^{2}}{a^{3}} ; q\right)_{2 j}}{\left(1-\frac{k^{2}}{a^{2}}\right)\left(\frac{k^{2} q^{2 n+2}}{a^{2}}, \frac{k q^{2-2 n}}{a^{2}} ; q^{2}\right)_{j}\left(\frac{k^{2}}{a^{3}}, q ; q\right)_{j}(a q ; q)_{2 j}} q^{j} \text {. }
\end{aligned}
$$




$$
\begin{gathered}
{ }_{10} \phi_{9}\left[\begin{array}{c}
\left.a, q \sqrt{a},-q \sqrt{a}, \frac{k}{a^{2}}, a \sqrt{\frac{a q}{k}},-a \sqrt{\frac{a q}{k}}, q^{-2 n},-q^{-2 n}, \sqrt{k} q^{2 n},-\sqrt{k} q^{2 n} ; q, \frac{-q^{2} a^{2}}{k}\right] \\
\sqrt{a},-\sqrt{a}, \frac{q a^{3}}{k}, \sqrt{\frac{q k}{a}},-\sqrt{\frac{q k}{a}}, q, \frac{a q^{1-2 n}}{\sqrt{k}},-\frac{a q^{1-2 n}}{\sqrt{k}}, a q^{2 n+1},-a q^{2 n+1}
\end{array}\right] \\
=\frac{\left(a^{2} q^{2}, \frac{a^{2}}{k} ; q^{2}\right)_{2 n}\left(\frac{-k}{a} ; q\right)_{4 n}\left(\frac{k q}{a^{2}}\right)^{2 n}}{\left(\frac{k}{a^{2}}, \frac{k^{2} q^{2}}{a^{2}} ; q^{2}\right)_{2 n}(-q a ; q)_{4 n}} \\
\times \sum_{j=0}^{n} \frac{\left(1-\frac{k^{2}}{a^{2}} q^{8 j}\right)\left(k q^{4 n}, q^{-4 n} ; q^{2}\right)_{2 j}\left(\frac{k}{a}, \frac{k^{2}}{a^{4}} ; q^{2}\right)_{j}}{\left(1-\frac{k^{2}}{a^{2}}\right)\left(\frac{k^{2} q^{4 n+2}}{a^{2}}, \frac{k q^{2-4 n}}{a^{2}} ; q^{2}\right)_{2 j}\left(q^{2}, \frac{q^{2} a^{3}}{k} ; q^{2}\right)_{j}} .
\end{gathered}
$$

\section{Bailey-Type Chains}

Just as Liu and Ma did in [7], we can let $k \rightarrow 0$ to get regular Bailey chains, but unfortunately none are new.

Corollary 9. If $\left(\alpha_{n}(a, q), \beta_{n}(a, q)\right)$ are a Bailey pair w.r.t. a, then so is $\left(\alpha_{n}^{\prime}(a, q), \beta_{n}^{\prime}(a, q)\right)$, where

$$
\begin{aligned}
& \alpha_{n}^{\prime}(a, q)=\frac{(1+a) q^{n}}{1+a q^{2 n}} \alpha_{n}\left(a^{2}, q^{2}\right) \\
& \beta_{n}^{\prime}(a, q)=\sum_{j=0}^{n} \frac{(-a ; q)_{2 j}}{\left(q^{2} ; q^{2}\right)_{n-j}} q^{j} \beta_{j}\left(a^{2}, q^{2}\right) .
\end{aligned}
$$

Proof. let $k \rightarrow 0$ in Corollary 4 and rearrange.

Remark: This is the Bailey chain (D4) of Bressoud, Ismail and Stanton in [5].

Corollary 10. If $\left(\alpha_{n}(a, q), \beta_{n}(a, q)\right)$ are a Bailey pair w.r.t. a, then so is $\left(\alpha_{n}^{\prime}(a, q), \beta_{n}^{\prime}(a, q)\right)$, where

$$
\begin{aligned}
& \alpha_{n}^{\prime}\left(a^{2}, q^{2}\right)=\alpha_{n}(a, q), \\
& \beta_{n}^{\prime}\left(a^{2}, q^{2}\right)=\frac{1}{(-a q ; q)_{2 n}} \sum_{j=0}^{n} \frac{(-1)^{n-j} q^{(n-j)^{2}}}{\left(q^{2} ; q^{2}\right)_{n-j}} \beta_{j}(a, q) .
\end{aligned}
$$

Proof. let $k \rightarrow 0$ in Corollary 7 and rearrange.

We note that is chain is also not new, being essentially the Bailey chain (D1) in [5].

The corollaries above and other results in the literature (for example the first WP-Bailey chain of Andrews in [1]) show that some Bailey chains may be "lifted" to WP-Bailey chains.

Is it possible to lift all Bailey chains to WP-Bailey chains? 


\section{REFERENCES}

[1] Andrews, George E. Bailey's transform, lemma, chains and tree. Special functions 2000: current perspective and future directions (Tempe, AZ), 1-22, NATO Sci. Ser. II Math. Phys. Chem., 30, Kluwer Acad. Publ., Dordrecht, 2001.

[2] Andrews, George; Berkovich, Alexander The WP-Bailey tree and its implications. J. London Math. Soc. (2) 66 (2002), no. 3, 529-549.

[3] Bressoud, David, The Rogers-Ramanujan identities. (solution to problem 74-12). SIAM Review. 23 (1981) 101-104.

[4] Bressoud, David, Some identities for terminating q-series. Math. Proc. Cambridge Philos. Soc. 89 (1981), no. 2, 211-223.

[5] Bressoud, D.; Ismail, M. E. H.; Stanton, D. Change of base in Bailey pairs. (English summary) Ramanujan J. 4 (2000), no. 4, 435-453.

[6] Gasper, George; Rahman, Mizan Basic hypergeometric series. With a foreword by Richard Askey. Second edition. Encyclopedia of Mathematics and its Applications, 96. Cambridge University Press, Cambridge, 2004. xxvi+428 pp.

[7] Q. Liu; X. Ma On the Characteristic Equation of Well-Poised Baily Chains - To appear.

[8] Mc Laughlin, James; Zimmer, Peter. Some Implications of the WP-Bailey Tree. Advances in Applied Mathematics Volume 43, Issue 2, August 2009, Pages 162-175.

[9] Singh, U. B. A note on a transformation of Bailey. Quart. J. Math. Oxford Ser. (2) 45 (1994), no. 177, 111-116.

[10] Spiridonov, V. P. An elliptic incarnation of the Bailey chain. Int. Math. Res. Not. 2002, no. 37, 1945-1977.

[11] Warnaar, S. O. Extensions of the well-poised and elliptic well-poised Bailey lemma. Indag. Math. (N.S.) 14 (2003), no. 3-4, 571-588.

Mathematics Department, Anderson Hall, West Chester University, West Chester, PA 19383

E-mail address: jmclaughl@wcupa.edu

Mathematics Department, Anderson Hall, West Chester University, West Chester, PA 19383

E-mail address: pzimmer@wcupa.edu 